\title{
Incontinência urinária e os critérios de fragilidade em idosos em atendimento ambulatorial
}

\author{
URINARY INCONTINENCE AND THE CRITERIA OF FRAILNESS AMONG THE \\ ELDERLY OUTPATIENTS
}

INCONTINENCIA URINARIA Y LOS CRITERIOS DE FRAGILIDAD EN ANCIANOS EN ATENCIÓN AMBULATORIA

\section{Vanessa Abreu da Silva ${ }^{1}$, Katia Lacerda de Souza ${ }^{2}$, Maria José D’Elboux ${ }^{3}$}

\section{RESUMO}

Este estudo teve como objetivos verificar a ocorrência de incontinência urinária (IU) e suas características em idosos pré-frágeis e frágeis atendidos em um ambulatório de geriatria, comparar a presença dos critérios de fragilidade entre os idosos com e sem IU e identificar entre os critérios de fragilidade a chance de risco para IU nesses idosos. Participaram do estudo 100 idosos, com média de idade 76,2 anos; 65 idosos relataram IU, $71,3 \%$ desses apresentavam três ou mais critérios de fragilidade. A ocorrência de IU foi superior nos idosos frágeis $(p=0,0011)$. A análise multivariada mostrou que os critérios lentidão $(O R=4,99)$ e exaustão $(O R=4,85)$ apresentaram relação estatisticamente significativa com IU. A ocorrência de IU foi elevada e aqueles idosos que apresentam lentidão têm chance risco quase cinco vezes maior de apresentar IU e os que referem exaustão tem chance de risco cinco vezes maior de IU quando comparados aos que não apresentam esses critérios.

\section{DESCRITORES}

Incontinência urinária

Idoso fragilizado

Saúde do idoso

Enfermagem geriátrica

\begin{abstract}
The objectives of this study were to verify the occurrence of urinary incontinence (UI) and its characteristics in pre-frail and frail elderly patients of a geriatrics outpatient clinic, compare the presence of frailness criteria among the elderly with and without UI and identify among the frailty criteria the chance of risk for UI among those elderly outpatients. Participants were 100 elderly individuals, with an average age of 76.2 years; 65 participants reported UI, $71.3 \%$ of which presented three or more frailness criteria. The occurrence of UI was greater in frail participants $(p=0.0011)$. Multivariate analysis showed that the criteria slowness ( $O R=4.99$ ) and exhaustion $(O R=4.85)$ has a statistically significant relation with UI. The occurrence of UI was high and participants who presented slowness have a risk almost five times greater to presenting UI while those reporting exhaustion have a risk five times greater for UI compared to those without these criteria.
\end{abstract}

\section{DESCRIPTORS}

Urinary incontinence

Frail elderly

Health of the elderly

Geriatric nursing

\section{RESUMEN}

Estudio que objetivó verificar la ocurrencia de incontinencia urinaria (IU) y sus características en ancianos pre-frágiles y frágiles atendidos en ambulatorio de geriatría, comparar la presencia de criterios de fragilidad entre ancianos con y sin IU e identificar entre los criterios de fragilidad la chance de riesgo para IU en estos ancianos. Participaron del estudio 100 ancianos, con edad media de 76,2 años; 65 ancianos refirieron IU, 71,3\% de ellos presentaban tres o más criterios de fragilidad. La ocurrencia de IU fue superior en ancianos frágiles ( $p=0,0011$ ). El análisis multivariado mostró que los criterios lentitud $(O R=4,99)$ y agotamiento $(O R=4,85)$ presentaron relación estadísticamente significativa con IU. La ocurrencia de IU fue elevada y aquellos ancianos con lentitud tienen chance de riesgo casi cinco veces mayor de presen$\operatorname{tar} I U, y$ los que refieren agotamiento tienen chance de riesgo cinco veces mayor de IU comparados con los que no presentan esos criterios.

\section{DESCRIPTORES \\ Incontinencia urinaria \\ Anciano frágil \\ Salud del anciano \\ Enfermería geriátrica}

${ }^{1}$ Enfermeira. Mestranda do Programa de Pós Graduação em Enfermagem da Faculdade de Ciências Médicas da Universidade Estadual de Campinas. Campinas, SP, Brasil. vanisabreu@hotmail.com ${ }^{2}$ Professora Associada do Departamento de Enfermagem da Faculdade de Ciências Médicas da Universidade Estadual de Campinas. Campinas, SP, Brasil. mariadio@uol.com.br ${ }^{3}$ Enfermeira. Residente do Programa de Residência em Cardiologia do Instituto Dante Pazzanese. São Paulo, SP, Brasil. bilu44ka44@yahoo.com.br

$\begin{aligned} & \text { Rev Esc Enferm USP } \text { Recebido: 02/03/2010 } \\ & \text { 2011; 45(3):672-8 } \text { Aprovado: 24/09/2010 } \\ & \text { www.ee.usp.br/reeuspl } \text { Português / Inglês } \\ & \text { www.scielo.br/reeusp }\end{aligned}$




\section{INTRODUÇÃO}

A temática incontinência urinária (IU) pouco tem sido investigada pelos profissionais de saúde e são escassos estudos sobre sua prevalência e incidência na literatura nacional(1), apesar de estudiosos a apontarem como um problema de saúde pública(2).

IU é definida como queixa de qualquer perda involuntária de urina $^{(3)}$ e é uma condição frequente na população em geral, acometendo cerca de $19 \%$ das mulheres e $10 \%$ dos homens com mais de 60 anos. Sua ocorrência aumenta exponencialmente com o avanço da idade frente a modificações funcionais e estruturais no sistema urinário e com o comprometimento da independência funcional. Portanto, estima-se que, com o crescimento da população de idosos, aumente consideravelmente a ocorrência de IU $\mathrm{IU}^{(2-3)}$.

Estudos internacionais mostram que a IU está presente em aproximadamente $30 \%$ dos idosos residentes na comunidade, 40,0 a 70,0\% nos hospitalizados e $50,0 \%$ nos institucionalizados. Representa um grande peso econômico, à medida que onera recursos financeiros de cuidado à saúde, além de aumentar expressivamente o risco de institucionalização, fragilidade, fraturas e depressão ${ }^{(3)}$

Além disso, a IU é considerada uma das mais importantes e recorrentes síndromes geriátricas $^{(4-7)}$, na medida em que muitos são os seus impactos na vida dos idosos. Merecem destaque as implicações negativas nas esferas emocional, social e econômica, tanto para o indivíduo incontinente, bem como para seus familiares e cuidadores ${ }^{(1)}$.

Evidências de pesquisas apontam a IU como um sinal precoce de pré-fragilidade e fragilidade no idoso. Também está associada ao risco aumentado de declínio funcional ${ }^{(8-9)}$.

O termo fragilidade, relacionado a idosos, raramente era citado antes de 1980. Referia-se ao idoso fraco, com défice cognitivo e físico, incapacitado, doente e que vivia em condições socioeconômicas desfavoráveis. Ainda há ausência de um consenso sobre a definição e a identificação da síndrome da fragilidade, o que representa um dos maiores obstáculos para as pesquisas clínicas ${ }^{(10)}$. Entretanto duas correntes de pesquisa se mostram mais consolidadas. Entre os estudiosos que pesquisam sobre a síndrome da fragilidade nos idosos, salienta-se nos Estados Unidos, o grupo de pesquisadores coordenado por Linda Fried(11), que propôs uma lista de critérios objetivos e mensuráveis que compõem o modelo unidimensional de fragilidade, considerando como prioritários os aspectos fisiopatológicos da síndrome. No Canadá, sobressaem-se os pesquisadores do grupo de Kenneth Rockwood, que consideram a fragilidade uma síndrome multidimensional, incluindo aspectos sociais, psicológicos, biológicos e econômicos, e acentuam a complexidade de sua etiologia, que compõe esse modelo, propondo a utilização de índices de fragilidade elaborados a partir da avaliação geriátrica abrangente ${ }^{(10)}$.

Uma das definições mais aceitas hoje é que a fragilidade é uma síndrome caracterizada pela diminuição da reserva energética e pela resistência reduzida aos estressores $^{(11)}$. Ocorrem três mudanças fisiológicas relacionadas à idade que são subjacentes à síndrome da fragilidade, conhecidas como tripé da fragilidade: alterações neuromusculares, desregulação do sistema endócrino e disfunção imunológica ${ }^{(11)}$.

Baseados nessas alterações, os pesquisadores do modelo unidimensional de fragilidade elaboraram um fenótipo de fragilidade composto por cinco critérios: perda de peso não-intencional no último ano, exaustão, lentidão, fraqueza muscular e baixo nível de atividade física ${ }^{(11)}$. Para ser considerado frágil, o idoso deve apresentar três ou mais critérios do fenótipo. A presença de um ou dois critérios caracteriza pré-fragilidade, dado grande risco de o idoso desenvolver a síndrome da fragilidade ${ }^{(12)}$.

Outrossim, ressaltam a importância da identificação precoce dos critérios de fragilidade, tendo em vista a prevenção de eventos adversos como descompensação de doenças crônicas, quedas, institucionalização, incapacidade e morte ${ }^{(12)}$. Além disso, estudos apontam que, na fase pré-frágil, a síndrome pode ser revertida ${ }^{(8)}$.

Na literatura pesquisada, observou-se escassez de trabalhos voltados ao estudo da presença de IU em idosos com critérios de fragilidade. Sendo assim, a relevância do presente estudo está na necessidade de verificar essa ocorrência, considerando que a IU pode ser um sinal de alarme para a síndrome da fragilidade, que por sua vez é um problema de saúde pública.

\section{OBJETIVOS}

- Verificar a ocorrência de IU e suas características em idosos pré-frágeis e frágeis atendidos em um ambulatório de geriatria.

- Comparar a presença dos critérios de fragilidade entre os idosos com e sem IU.

- Identificar entre os critérios de fragilidade a chance de risco para a ocorrência de IU nesses idosos.

\section{MÉTODO}

Trata-se de um estudo quantitativo, descritivo e de corte transversal, realizado no Ambulatório de Geriatria do Hos- 
pital de Clínicas (HC) da Universidade Estadual de Campinas (Unicamp). O Ambulatório situa-se no terceiro andar do hospital e, às quintas-feiras no período vespertino, sua equipe multiprofissional presta atendimento aos idosos com idade $\geq 80$ anos ou acima de 60 anos e com dependência física ou cognitiva.

A coleta de dados foi realizada de fevereiro a agosto de 2009. A amostra dessa pesquisa foi de conveniência, compreendida por 100 idosos atendidos no Ambulatório e que atenderam aos seguintes critérios de inclusão: idade mínima de 60 anos; concordância em participar do estudo, com assinatura do Termo de Consentimento Livre e Esclarecido; em condições de comunicação; pontuação no MiniExame do Estado Mental (MEEM) $\geq 13$ (para analfabetos), $\geq 18$ ( 1 a 7 anos de escolaridade), $\geq 26$ (igual ou superior a 8 anos)(13), e que apresentaram pelo menos um, entre os cinco critérios de fragilidade do modelo unidimensional de fragilidade ${ }^{(11)}$. Como critérios de exclusão foram considerados: idosos que não apresentaram condições de comunicação oral, presença de défice cognitivo ou pontuações inferiores no MEEM citadas nos critérios de inclusão e ausência de critério de fragilidade adotado no estudo.

Para a avaliação da fragilidade, foi utilizado o modelo unidimensional de fragilidade ${ }^{(11)}$, composto por cinco critérios, aqui já descritos. Para isso, foi necessário efetuar adaptação em três deles, pois no período em que o instrumento foi elaborado (2002), para a realização de um projeto temático, não foi possível obter na literatura informações pormenorizadas sobre o procedimento de avaliação de cada critério. Assim, a exemplo de outros estudos encontrados na literatura, tentou-se ajustar os critérios deste estudo aos critérios publicados posteriormente por pesquisadores deste modelo, conforme segue:

- Perda de peso involuntária no último ano: em caso de perda de peso superior a 4,5 quilos ou mais de $10 \%$ do peso corporal no último ano, atribuiu-se pontuação positiva para este critério, sendo que no modelo original é considerada perda superior a $5 \%$ do peso corporal;

- Exaustão: avaliada por duas questões da escala de rastreamento de depressão The Centerfor Epidemiologic Studies - Depression (CES-D) para detecção do número de vezes, na última semana, o entrevistado sentiu que teve que fazer esforço para dar conta das tarefas habituais e que não conseguiu levar adiante suas coisas ${ }^{(14)}$. Se a resposta para ambas for afirmativa por três dias ou mais da semana anterior, atribuiu-se pontuação positiva, conforme o modelo original;

- Lentidão: avaliada pela velocidade da marcha diminuída, ou seja, tempo cronometrado para percorrer duas vezes uma distância de 4,0 metros, selecionando o menor tempo, ajustado por sexo e altura. Foram pontuados os resultados maiores ou iguais a 7 segundos para homens com altura menor ou igual a $173 \mathrm{~cm}$ e 6 segundos para homens com altura menor que $173 \mathrm{~cm}$; já para as mulheres resultados maiores ou iguais a 7 segundos, para aquelas com altura menor ou igual a $159 \mathrm{~cm}$, e maiores que 6 se- gundos, para aquelas com altura maior que $159 \mathrm{~cm}$; no modelo original a distância utilizada é 4,5 metros;

- Fraqueza muscular: avaliada por meio da força de preensão palmar, medida por meio do dinamômetro manual portátil na mão dominante, com o idoso na posição ortostática. Foi utilizado o maior valor de três medidas, realizadas em um intervalo aproximado de 5 minutos entre elas. Os resultados foram estratificados por sexo e índice de massa corpórea (IMC). Atribuí-se pontuação de acordo com os critérios apresentados no Quadro 1.

Quadro 1 - Critérios de fragilidade para fraqueza muscular - força de preensão palmar - Campinas - 2010

\begin{tabular}{|c|c|}
\hline IMC em $\mathbf{~ K g} / \mathbf{m}^{\mathbf{2}}$ & Pontos de corte para fragilidade em Kgf \\
\hline Homens & $\leq 229$ \\
$\leq 24$ & $\leq 30$ \\
24,1 a 26 & $\leq 31$ \\
26,1 a 28 & $\leq 32$ \\
$>28$ & \\
\hline Mulheres & $\leq 17$ \\
$\leq 23$ & $\leq 17,3$ \\
23,1 a 26 & $\leq 18$ \\
26,1 a 29 & $\leq 21$ \\
$>29$ & \\
\hline
\end{tabular}

Fonte: Adaptado do modelo unidimensional de fragilidade ${ }^{(11)}$.

- Baixo nível de atividade física: perguntou-se ao participante se praticava atividade física e a sua frequência. Em caso de resposta negativa ou de frequência uma ou duas vezes por semana, pontuou-se para fragilidade. No modelo original, é realizada avaliação do dispêndio energético semanal, baseado no auto-relato de atividades e exercícios físicos avaliados pelo Minnesota Leisure Time Activities Questionnaire e ajustado de acordo com o sexo.

A coleta de dados foi feita por meio de entrevista estruturada, utilizando-se os seguintes instrumentos: 1) Caracterização sociodemográfica, para a coleta de informações relacionadas à caracterização da amostra. Para avaliar a sua adequação à proposta de investigação, o instrumento foi submetido à validade de conteúdo e avaliado por um comitê de quatro juízes experts nas áreas de Geriatria e Gerontologia, bem como na área de IU; 2) O International Consultation on Incontinence Questionnaire-Short Form (ICIQ-SF), para a avaliação da IU, é um instrumento adaptado e validado para a língua portuguesa do Brasil(15). O ICIQ-SF é composto por seis questões, as quais avaliam a frequência, a gravidade da perda urinária, e interferência da IU na vida diária, além de uma seqüência de oito itens autodiagnósticos, relacionadas às causas ou situações de IU, que não são pontuados. A soma dos escores (ICIQ Escore) das questões três, quatro e cinco varia de 0 a 21. A interferência da IU na vida diária é definida de acordo com o escore da questão 5 : (0) nada; (1-3) leve; (4-6) moderado; (7-9) grave; (10) muito grave. Para ser considerado incontinente, o idoso deve apresentar escore maior ou igual a três e, quanto mais elevado o escore total, maior é a gravidade da IU ${ }^{(15)}$. 
Após a avaliação dos idosos quanto à fragilidade, conforme os critérios adotados neste estudo, foram obtidos dois grupos: um com 41 (41,0\%) sujeitos com um ou dois critérios, ou seja, considerados pré-frágeis, e outro composto por $59(59,0 \%)$ sujeitos com três ou mais critérios, identificados como frágeis.

As análises estatísticas utilizadas nesse estudo estão descritas a seguir:

- Análise descritiva para as variáveis sociodemográficas, para os escores do ICIQ-SF e critérios de fragilidade;

- Coeficiente Alfa de Cronbach para avaliação da consistência interna do instrumento ICIQ-SF - este coeficiente é utilizado para verificar a homogeneidade dos itens, ou seja, sua acurácia. O alfa de Cronbach do ICIQ-SF foi considerado satisfatório com valor de 0.84 para a questão três, 0,86 para a questão quatro, 0,88 para a questão cinco e o alfa Cronbach ponderado foi de 0,89 (Critérios satisfatórios $\alpha=0,70)^{(16)}$.

- Teste Qui-Quadrado ou Exato de Fisher para comparar os idosos com e sem IU e os critérios de fragilidade.

- Análise de Regressão Logística Univariada e Multivariada para estudar a relação entre IU e os critérios de fragilidade.

O nível de significância estatística adotado para os testes foi $5 \%$, ou seja, $p<0,05$.

Este estudo integra o projeto temático intitulado Qualidade de vida em idosos: indicadores de fragilidade e de bem-estar subjetivo, que foi submetido e aprovado pelo Comitê de Ética em Pesquisas (CEP) da FCM-UNICAMP (Parecer CEP 210/2003) bem como o adendo referente à avaliação de IU (Parecer CEP 240/2003).

\section{RESULTADOS}

No período de coleta de dados, foram atendidos 143 idosos no Ambulatório de Geriatria, dos quais 100 deles corresponderam aos critérios de inclusão. Conforme apresentado na Tabela 1, as características predominantes da amostra foram: sexo feminino (74\%); idade superior a 70 anos $(78,0 \%)$ com média de 76,6 anos $( \pm 7,8)$; procedência do município de Campinas (54\%) e baixa escolaridade $(49,0 \%)$.

Referiram perda involuntária de urina $65,0 \%$ dos idosos entrevistados, ou seja, obtiveram escore maior ou igual a três no ICIQ-SF, o que significa que o idoso refere perda urinária uma vez por semana ou menos e em pequena quantidade. Do total de 65 idosos com IU, 40 (61,4\%) relataram perda de urina diversas vezes ao dia e em pequena quantidade. O escore referente ao impacto da IU na vida diária dos idosos, avaliado pela questão cinco do ICIQ-SF, variou de zero a 10 (média 4,85) e quase metade dos idosos $(49,2 \%)$ a consideraram muito grave (Tabela 2 ).
Tabela 1 - Caracterização sociodemográfica e de fragilidade dos idosos do estudo - Campinas - 2010

\begin{tabular}{lrrrr}
\hline Características & N & $\%$ & $\begin{array}{c}\text { Variação } \\
\text { Mínimo - } \\
\text { máximo }\end{array}$ & $\begin{array}{c}\text { Média } \\
\text { (Desvio } \\
\text { padrão) }\end{array}$ \\
\hline $\begin{array}{l}\text { Sexo } \\
\quad \text { Masculino }\end{array}$ & 26 & 26,0 & & \\
$\quad$ Feminino & 74 & 74,0 & & \\
\hline Faixa Etária & & & $60-93$ & $76,6( \pm 7,8)$ \\
\hline Procedência & & & & \\
Campinas & 55 & 55,0 & & \\
Outros municípios & 45 & 45,0 & & \\
\hline Escolaridade & & & & \\
$\quad \leq 4$ anos & 49 & 49,0 & & \\
$>4$ anos & 9 & 9,0 & & \\
Analfabetos/ Sabe & 42 & 42,0 & & \\
ler e escrever & & & & \\
\hline Fragilidade & & & & \\
$1-2$ critérios & 41 & 41,0 & & \\
$3-5$ critérios & 59 & 59,0 & & \\
\hline
\end{tabular}

Nota: $(n=100)$

Tabela 2 - Distribuição dos idosos que referiram perda urinária de acordo com as questões do ICIQ-SF - Campinas - 2010

\begin{tabular}{|c|c|c|}
\hline ICIQ-SF & $\mathbf{N}$ & $\%$ \\
\hline \multicolumn{3}{|l|}{ Frequência de perda urina } \\
\hline 1x por semana ou menos & 4 & 6,0 \\
\hline $2 \mathrm{x}$ ou $3 \mathrm{x}$ por semana & 11 & 17,0 \\
\hline $1 \mathrm{x}$ por dia & 7 & 11,0 \\
\hline Diversas vezes ao dia & 40 & 61,5 \\
\hline Tempo todo & 3 & 4,5 \\
\hline \multicolumn{3}{|l|}{ Quantidade de perda urinária } \\
\hline Pequena quantidade & 40 & 61,5 \\
\hline Moderada quantidade & 13 & 20,0 \\
\hline Grande quantidade & 12 & 18,5 \\
\hline \multicolumn{3}{|l|}{ Interferência na vida diária } \\
\hline Nada $(0)$ & 10 & 15,4 \\
\hline Leve (1-3) & 6 & 9,2 \\
\hline Moderado (4-6) & 6 & 9,2 \\
\hline Grave (7-9) & 11 & 17,0 \\
\hline Muito grave (10) & 32 & 49,2 \\
\hline \multicolumn{3}{|c|}{ Causas ou situações de perda involuntária de urina* } \\
\hline Antes de chegar ao banheiro & 50 & 76,9 \\
\hline Ao tossir ou espirrar & 37 & 56,9 \\
\hline Ao dormir & 11 & 16,9 \\
\hline Ao realizar atividades físicas & 12 & 18,4 \\
\hline Ao se vestir após urinar & 16 & 24,6 \\
\hline Sem razão óbvia & 9 & 13,8 \\
\hline O tempo todo & 6 & 9,2 \\
\hline
\end{tabular}

* O idoso poderia responder mais de uma alternativa. Nota: $(n=65)$

As principais causas ou situações de perda urinária apontadas pelos 65 idosos foram antes de chegar ao banheiro (50\%) e perda ao tossir ou espirrar (37\%).

Na comparação entre os idosos com e sem IU e os critérios de fragilidade, houve diferença estatisticamente significa- 
tiva $(p=0,001)$ quanto ao número de critérios, ou seja, $62,8 \%$ dos idosos sem IU apresentaram um a dois critérios (pré-frágeis), enquanto, 70,7\% dos idosos com IU apresentaram três ou mais critérios (frágeis). Assim, verificou-se que os idosos estudados com IU pontuaram maior número de critérios de fragilidade, quando comparados aos idosos sem IU.

Em relação a cada critério adotado no estudo, todos apresentaram diferença significativa entre os idosos com e sem IU, com exceção do critério perda de peso involuntária no último ano. Os critérios baixa atividade física e lentidão foram os mais pontuados pelos idosos com e sem IU, 88,0\% e 65,0\% respectivamente. Entretanto, houve maior número de idosos com IU, diferença esta estatisticamente significativa (Tabela 3).

Tabela 3 - Comparação entre idosos com IU (n=65) e sem IU ( $n=35)$ de acordo com os critérios de fragilidade - Campinas - 2010

\begin{tabular}{|c|c|c|c|c|}
\hline $\begin{array}{l}\text { Critérios de } \\
\text { Fragilidade }\end{array}$ & $\begin{array}{c}\text { C Com IU } \\
\text { N }(\%)\end{array}$ & $\begin{array}{l}\text { Sem IU } \\
\text { N }(\%)\end{array}$ & $\begin{array}{l}\text { Total } \\
\text { N (\%) }\end{array}$ & p-valor* \\
\hline \multicolumn{5}{|l|}{ Número de critérios } \\
\hline $\begin{array}{l}1 \text { ou } 2 \text { critérios } \\
\text { (Pré-frágeis) }\end{array}$ & $19(29,2)$ & $22(62,8)$ & $41(41,0)$ & \\
\hline $\begin{array}{l}3 \text { ou mais critérios } \\
\text { (Frágeis) }\end{array}$ & $46(70,8)$ & $13(37,2)$ & $59(59,0)$ & 0,0011 \\
\hline \multicolumn{5}{|l|}{ Critérios $* * *$} \\
\hline $\begin{array}{l}\text { Perda de peso } \\
\text { involuntária }\end{array}$ & $18(27,7)$ & $10(28,6)$ & $28(28,0)$ & $0,9256^{*}$ \\
\hline Fraqueza muscular & $40(61,5)$ & $13(37,1)$ & $53(53,0)$ & $0,0197^{*}$ \\
\hline $\begin{array}{l}\text { Velocidade da marcha } \\
\text { diminuída }\end{array}$ & $49(75,3)$ & $15(42,8)$ & $65(65,0)$ & $0,0012^{*}$ \\
\hline Exaustão & $42(64,6)$ & $11(31,4)$ & $53(53,0)$ & $0,0015^{*}$ \\
\hline Baixa atividade física & $61(93,8)$ & $27(77,1)$ & $88(88,0)$ & $0,0223 * *$ \\
\hline
\end{tabular}

* teste Qui-Quadrado ** teste Exato de Fisher *** O idoso poderia apresentar mais de um critério. Nota: $(n=100)$

Como houve diferença estatística na comparação entre os grupos com e sem IU e os critérios de fragilidade foi realizada Análise de Regressão Logística Univariada e Múltipla para estudar a relação entre IU e os critérios de fragilidade, conforme apresentados nas Tabelas 4 e 5.

Tabela 4 - Resultados da Análise de Regressão Logística Univariada para IU em idosos frágeis e pré-frágeis - Campinas - 2010

\begin{tabular}{lcccc}
\hline Variável & Categorias & p-valor & OR* & IC 95\% OR** \\
\hline $\begin{array}{l}\text { Perda de peso } \\
\text { involuntária }\end{array}$ & Não (ref.) & --- & 1,00 & --- \\
Fraqueza muscular & Sim & 0,925 & 0,96 & $0,38-2,39$ \\
& Não (ref.) & --- & 1,00 & --- \\
Lentidão & Sim & 0,021 & 2,71 & $1,16-6,33$ \\
& Não (ref.) & --- & 1,00 & --- \\
Exaustão & Sim & 0,002 & 4,08 & $1,70-9,80$ \\
& Não (ref.) & --- & 1,00 & --- \\
Baixa atividade & Sim & 0,002 & 3,98 & $1,66-9,57$ \\
física & Não (ref.) & --- & 1,00 & --- \\
\hline
\end{tabular}

* OR (Odds Ratio) = Razão de risco para incontinência urinária; ( $\mathrm{n}=35$ sem incontinência e n=65 com incontinência). **IC 95\% OR = Intervalo de 95\% de confiança para a razão de risco. Ref.: nível de referência. Nota: $(n=100)$
Tabela 5 - Resultados da Análise de Regressão Logística Multivariada para IU em idosos frágeis e pré-frágeis - Campinas - 2010

\begin{tabular}{lcccc}
\hline $\begin{array}{l}\text { Variáveis } \\
\text { Selecionadas }\end{array}$ & Categorias & p-valor & OR* & IC 95\% OR** \\
\hline Lentidão & Não (ref.) & --- & 1,00 & --- \\
& Sim & $\mathbf{0 , 0 0 1}$ & 4,99 & $1,90-13,12$ \\
\multirow{2}{*}{ Exaustão } & Não (ref.) & --- & 1,00 & --- \\
& Sim & $\mathbf{0 , 0 0 1}$ & 4,85 & $1,85-12,73$ \\
\hline
\end{tabular}

* OR (Odds Ratio) = Razão de risco para incontinência urinária; ( $\mathrm{n}=35$ sem incontinência e n=65 com incontinência). **IC 95\% OR = Intervalo de 95\% de confiança para a razão de risco. Critério Stepwise de seleção de variáveis. Nota: $(n=100)$

Pelos resultados da Análise Multivariada com critério Stepwise de seleção de variáveis, verificou-se que os critérios lentidão e exaustão foram estatisticamente significativos, ou seja, os idosos que apresentavam velocidade de marcha diminuída tinham risco quase cinco vezes maior de IU comparados com os que não apresentavam $(\mathrm{OR}=4,99)$, e os idosos que apresentavam exaustão tinha risco aproximado cinco vezes maior de IU comparados com os que não apresentavam (OR=4,85).

\section{DISCUSSÃO}

A ocorrência de IU foi de $65 \%$ na amostra idosa investigada, valor esse muito superior ao descrito na literatura nacional e internacional, especialmente entre idosos residentes na comunidade. Entretanto, foi mais próximo aos resultados de estudos com idosos institucionalizados. No Brasil, destaca-se o estudo realizado no município de São Paulo com 2.143 idosos da comunidade em que os pesquisadores encontraram uma ocorrência de IU de 11,8\% em homens e $26,2 \%$ em mulheres ${ }^{(17)}$. Entre idosos com demência, considerados frágeis pela essência de seu diagnóstico, em acompanhamento ambulatorial a ocorrência de IU encontrada foi de $33,3 \%{ }^{(18)}$. Por outro lado, em amostras de idosos com maior vulnerabilidade, ou melhor, em situações de seguimento assistencial à saúde ou institucionalizados, situações que procedem a síndrome da fragilidade, esses valores aumentam ${ }^{(4)}$. A IU em idosos frágeis constitui um modelo sindrômico com interação de múltiplos fatores de risco, tais como alterações psicológicas e cognitivas relacionadas à idade e comorbidades ${ }^{(9)}$.

No que diz respeito às características da IU, não há consenso na literatura sobre a frequência e a quantidade da perda urinária, uma vez que é consequente a diversos fatores, tais como o tipo de IU, o estado de saúde do sujeito, comorbidades, grau de dependência funcional, entre outros. No presente estudo, $61,5 \%$ referiram perda de urina diversas vezes ao dia em pequena quantidade. Resultados semelhantes foram obtidos em estudo com idosas institucionalizadas. Em investigação internacional, os autores descreveram que $31,8 \%$ das idosas perdiam urina diversas vezes ao $\mathrm{dia}^{(19)}$. Outro estudo realizado em Hong Kong com 148 idosos em acompanhamento ambulatorial mostrou que $16,7 \%$ dos idosos referiam perder urina menos 
do que três vezes por semana, $12,5 \%$ diariamente e $4,2 \%$ de três a seis vezes por semana ${ }^{(18)}$.

O impacto da IU na vida diária foi considerado pela maioria dos participantes como muito grave, apesar do predomínio de relatos de perda em pequena quantidade de urina. $\mathrm{O}$ mesmo também encontrado em estudo internacional onde $81,0 \%$ dos idosos relataram grande impacto na qualidade de vida decorrente das perdas urinárias ${ }^{(18)}$. Esta perda causa uma interferência negativa na vida diária dos idosos, vinculada à perda da independência para frequentar festas de família, igreja, mercado, entre outros locais, frente ao medo e vergonha de perder urina e exalar odores característicos ${ }^{(20)}$.

As situações mais freqüentes em que ocorre a perda urinária, antes de chegar ao banheiro e aos esforços, sugerem a presença de IU tipo urge-incontinência e IU aos esforços na maioria dos idosos entrevistados, confirmando achados nacionais e internacionais quanto aos tipos mais comuns de IU na velhice. A principal causa da IU tipo urgeincontinência está relacionada ao aumento da frequência de hiperatividade detrusora, de acordo com estudos sobre a avaliação urodinâmica em idosos ${ }^{(18)}$.

Os resultados revelaram que, em idosos pré-frágeis e frágeis, há expressivo aparecimento de IU, uma vez que, entre os 100 idosos com critérios de fragilidade, $65 \%$ apresentaram IU. Destaca-se, ainda, que a presença de IU foi mais comum entre os idosos com três ou mais critérios de fragilidade, quando comparados com aqueles que apresentaram até dois critérios. Isto sugere que, na medida em que aumenta o número de critérios de fragilidade, há maior risco para o desenvolvimento de IU.

Entre os critérios de fragilidade, a Análise de Regressão Logística Multivariada mostrou significância estatística entre os critérios lentidão e exaustão, ou seja, os idosos com lentidão têm risco cinco vezes maior de IU comparados com os que não apresentam, e os idosos com exaustão têm risco 4.9 vezes maior de IU comparados com os que não apresentam. Na literatura pesquisada, são escassos os estudos que descrevem a presença de IU em idosos com critérios de fragilidade, porém o tripé da fragilidade ${ }^{(11)}$ aponta que as alterações neuromusculares relacionadas à idade são subjacentes à síndrome da fragilidade ${ }^{(8,12)}$ e sabe-se que a lentidão está associada com alterações de mobilidade que podem dificultar o acesso do idoso ao banheiro e favorecer a ocorrência de IU, especialmente em caso de urgeincontinência ${ }^{(11)}$. Muitos estudos apresentaram associação entre IU e mobilidade em idosos ${ }^{(17-18)}$.

Além disso, a lentidão tem estreita relação com atividade física, podendo ser consequência da redução da atividade física ou até mesmo sua causa. Estudos indicam que redução de atividade física pode ser fator de início da IU em idosos frágeis ${ }^{(8)}$, portanto a manutenção da atividade física pode retardar o início da IU nessa população, uma vez que essa redução já está estabelecida é necessário realizar reabilitação física no sentido de melhorar essa condição e, consequentemente, prevenir o desenvolvimento de IU. A esse respeito, destaca-se a investigação realizada no Japão em que os autores obtiveram significativa taxa de cura de IU após melhora da velocidade de marcha em idosos com IU submetidos a tratamento com exercícios físicos ${ }^{(21)}$.

Quanto à exaustão, acredita-se que esta condição pode contribuir para IU na medida em que pode provocar desânimo e falta de motivação para ir ao banheiro.

Os resultados deste estudo enfatizam necessidade de implementação de medidas práticas que melhorem a força muscular e, consequentemente, a lentidão, tais como exercícios físicos. Além disso, medidas como fortalecimento da musculatura do soalho pélvico e alterações comportamentais relacionadas à alimentação, ingesta hídrica e aos hábitos de eliminação urinária, são estratégias que irão favorecer a redução da IU a priori urge-incontinência e IU aos esforços, tipos de maior ocorrência nesse estudo.

Assim como a fragilidade pode resultar a morte, a IU está associada a aumento do risco de mortalidade em idosos $^{(22)}$. Portanto, a Sociedade Internacional de Continência (International Continence Society) recomenda que seja realizada investigação de IU em todos idosos com critérios de fragilidade ${ }^{(9)}$.

Tendo em vista os resultados obtidos, destaca-se a contribuição do estudo no sentido de subsidiar o desenvolvimento de estratégias de intervenção pela equipe de saúde, com a finalidade de recuperação e da prevenção de agravos à saúde. Os idosos pré-frágeis são menos incontinentes, o que remete para a necessidade de programar ações precoces, visando à prevenção da IU e da síndrome da fragilidade no idoso. Com a regressão desse quadro, consequentemente, será possível obter redução do ônus econômico, social e psicológico.

\section{CONCLUSÃO}

A IU nos idosos deste estudo foi elevada (65\%) e a queixa mais frequente foi de perda urinária em pequena quantidade e diversas vezes ao dia. Com exceção do critério perda de peso involuntária, todos os critérios de fragilidade apresentaram relação estatisticamente significativa com IU e, entre os idosos com critérios de fragilidade, aqueles que apresentam lentidão, têm risco quase cinco vezes maior de apresentar IU e os que referem exaustão têm risco aproximadamente cinco vezes maior de IU quando comparados aos idosos que não tiveram esses critérios.

Este estudo possui algumas limitações, dentre elas o fato de que não foi possível avaliar detalhadamente o gasto energético semanal por meio do Minnesota Leisure Time Activities Questionnaire. Por se tratar de um estudo transversal, seria interessante realizar um estudo longitudinal para acompanhar idosos com IU a fim de verificar se estes desenvolvem a síndrome da fragilidade.

A avaliação da IU foi efetuada pelo instrumento ICIQSF que, apesar de ser um instrumento reconhecido e am- 
plamente utilizado na literatura para esse propósito, a avaliação da IU não deve-se limitar a ele. Outros aspectos devem ser considerados tais, como anamnese, exame físico geral e específico, do ponto de vista neurológico e geniturinário, avaliação do diário miccional e avaliação urodinâmica, além de outras avaliações específicas para IU.

\section{REFERÊNCIAS}

1. Silva APM, Santos VLCG. Prevalência da incontinência urinária em adultos e idosos hospitalizados. Rev Esc Enferm USP. 2005;39(1):36-45.

2. Santos CRSS, Santos VLCGS. Epidemiologia das incontinências urinária e anal combinadas. Acta Paul Enferm. 2009;22(3):328-30.

3. Haylen BT, Ridder D, Freeman RM, Swit SE, Berghmans B, Lee J, et al. An International Urogynecological Association (IUGA)/ International Continence Society (ICS) joint report on the terminology for female pelvic floor dysfunction. Neurourol Urodyn. 2010;29(1):4-20.

4. Du Moulin MF, Hamers JPH, Ambergen AW, Halfens RJG. Urinary incontinence in older adults receiving home care diagnosis and strategies. Scand J Caring Sci. 2009;23(2):222-30.

5. Inouye SK, Studenski S, Tinetti ME, Kuchel GA. Geriatric syndromes: clinical, researche, and policy implications of a core geriatrics concept. Am Geriatr Soc. 2007;55(5):780-91.

6. Padrós J, Peris T, Salva A, Denkinger MD, Coll-Planas L. Evaluation of a urinary incontinence unit for communitydwelling older adults in Barcelona: improvement of the perceived and severity of urinary incontinence. Z Gerontol Geriatr. 2008;41(4):291-7.

7. Lee PG, Cigolle C, Blaum C. The co-ocorrence of chronic diseases and geriatric syndromes: the health and retirement study. J Am Geriatr Soc. 2009;57(3):511-6.

8. Coll-Planas L, Denkinger MD, Nikolaus T. Relationship of urinary incontinence and late-life disability: implications for clinical work and research in geriatrics. Z Gerontol Geriatr. 2008;41(4):283-90.

9. DuBeau CE, Kuchel GA, Jhonson T 2nd, Palmer MH, Wagg A. Incontinence in frail elderly: report from the $4^{\text {th }}$ International Consultation on Incontinence. Neurourol Urodyn. 2010;29(1):165-78.

10. Rockwood K, Hogan DB, MacKnigh D. Conceptualisation and measurement of frailty in elderly people. Drugs Aging. 2000;17(4):295-302.

11. Fried $L P$, Tangen $C M$, Walston J, Newman $A B$, Hirsch $C$, Gottdiner J, et al. Frailty in older adults: evidence for a phenotype. J Gerontol. 2001;5(3):146-51.
12. Ahmed N, Mandel R, Fain M. Frailty: an emerging geriatric syndrome. Am J Med. 2007;120(9):748-53.

13. Bertolucci PHF, Brucki SMD, Capamacci S, Juliano Y. O miniexame do estado mental em uma população geral: impacto da escolaridade. Arq Neuropsiquiatr.1994;52(1):1-7.

14. Batistoni SS, Neri AL, Cupertino AP. Validade da escala de depressão do Center for Epidemiological Studies entre idosos brasileiros. Rev Saúde Pública. 2007;41(4):598-605.

15. Tamanini JTN, Dambros M, Dáncona CAL, Palma PCR, Netto Junior NR. Validação para o português do "International Consultation on Incontinence Questionnaire - Short Form" (ICIQ-SF). Rev Saúde Publica. 2004;38(3):438-44.

16. Hatcher L. A step-by-step approach to using SAS System for factor analysis and structural equation modeling. Cary (NC): SAS Institute; 1994.

17. Tamanini JT, Lebrão ML, Duarte YAO, Santos JLF, Laurenti R. Analysis of the prevalence and factors associeted with urinary incontinence among elderly people in the Municipality of São Paulo, Brazil: SABE Study (Health, Wellneing and Aging). Cad Saúde Pública. 2009;25(8):1756-62.

18. Miu DK, Lau S, Szeto SS. Etiology and predictors of urinary incontinence and its effects on quality of life. Geriatr Gerontol Int. 2010;10(2):177-82.

19. Lazari ICF, Lojudice DC, Marota AG. Avaliação da qualidade de vida de idosas com incontinência urinária: idosas institucionalizadas em instituição de longa permanência. Rev Bras Geriatr Gerontol. 2009;12(1):103-12.

20. Phelan S, Grodstein F, Brown JS. Clinical research in diabetes and urinary incontinence: what we know and need to know. J Urol. 2009;182(6 Suppl):S14-7.

21. Kim H, Suzuki T, Yoshida Y, Yoshida H. Effectiveness of multidimensional exercises for the treatment of stress urinary incontinence in elderly community-dwelling Japanese women: a randomized, controlled, crossover trial. J Am Geriatr Soc. 2007; 55(12):1932-9.

22. Jhonson TM, Bernard SL, Kincade JE, Defries GH. Urinary incontinence and risk of death among community-living elderly people: results from the National Survey on Self-care and Aging. J Aging Health. 2000;12(1):24-46.
Correspondência: Vanessa de Abreu Silva

Rua Deoclésio Camara Mattos, 570 - Vila Carminha CEP 13045425 - Campinas, SP, Brasil 